\title{
Effect of intermittent and continuous caloric restriction on Sirtuin1 concentration depends on sex and body mass index
}

\author{
Trine B. Opstad a,b,*, Tine Sundfør ${ }^{\text {c }}$, Serena Tonstad ${ }^{\text {c }}$, Ingebjørg Seljeflot ${ }^{\mathrm{a}, \mathrm{b}}$ \\ ${ }^{a}$ Center for Clinical Heart Research, Department of Cardiology, Oslo University Hospital Ullevål, Oslo, Norway \\ ${ }^{b}$ Faculty of Medicine, University of Oslo, Norway \\ ${ }^{c}$ Section of Preventive Cardiology, Department of Endocrinology, Morbid Obesity and Preventive Medicine, Oslo University Hospital Aker, Oslo, Norway
}

Received 21 January 2021; received in revised form 8 March 2021; accepted 9 March 2021

Handling Editor: A. Siani

Available online 19 March 2021

\section{KEYWORDS \\ Sirtuin1; \\ Caloric restriction; \\ BMI; \\ Sex}

\begin{abstract}
Background \& aims: The favorable effect of caloric restriction (CR) on health span is well known and partly mediated by the sirtuin system. Sirtuin1, a regulator of energy homeostasis in response to nutrient availability, is activated by CR. We therefore investigated effects of two different CR regimens on Sirtuin1 concentrations.

Methods $\mathcal{E}$ results: The study included 112 abdominally obese subjects, randomized to intermittent or continuous $\mathrm{CR}$ for 1 year. Blood samples and anthropometric measures were collected at baseline and after 12 months. Sirtuin1 concentrations were measured by ELISA. Sirtuin1 correlated significantly to BMI at baseline $(r=.232, \mathrm{p}=0.019)$. Mean reduction in body-weight was 8.0 and $9.0 \mathrm{~kg}$ after intermittent and continuous CR, respectively. After 1 year, no significant between-group differences in Sirtuin1 levels were observed according to regimen $(p=0.98)$ and $\operatorname{sex}(p=0.41)$. An increase in median Sirtuin 1 concentrations $(\mathrm{pg} / \mathrm{mL})[25,75$ percentiles] from baseline was observed after intermittent $C R$ in the total population $(884[624,1285]$ vs.762 $[530,1135] ; p=0.041)$, most marked in men $(820[623,1250]$ vs. $633[524,926] ; p=0.016)$. Improvement in BMI after 1 year correlated to Sirtuin 1 changes, but varied according to sex. In women, Spearman's rho $=.298, \mathrm{p}=0.034$, with stronger correlation in the intermittent CR group $(\mathrm{r}=.424, \mathrm{p}=0.049)$. In men, there was an inverse relation to Sirtuin1 changes, only in the intermittent CR group $(r=-.396, p=0.045)$. Conclusions: Effects on Sirtuin1 concentrations after 1 year of $\mathrm{CR}$ are sex and BMI-related. Intermittent $\mathrm{CR}$ regimen affected Sirtuin 1 to a stronger extent than continuous CR, suggesting individualized dietary intervention.

() 2021 The Author(s). Published by Elsevier B.V. on behalf of The Italian Diabetes Society, the Italian Society for the Study of Atherosclerosis, the Italian Society of Human Nutrition and the Department of Clinical Medicine and Surgery, Federico II University. This is an open access article under the CC BY license (http://creativecommons.org/licenses/by/4.0/).
\end{abstract}

Abbreviations: BMI, body mass index; CCR, continuous caloric restriction; CR, caloric restriction; CVD, cardiovascular disease; ICR, intermittent caloric restriction; IL, interleukin; NAD, nicotinamide adenine dinucleotide; SIRT1, Sirtuin1; STACs, sirtuin-activating compounds.

* Corresponding author. Center for Clinical Heart Research, Department of Cardiology, Oslo University Hospital, Ullevål, Kirkeveien 166, Pb 4950 Nydalen, N-0240 Oslo, Norway.

E-mail address: trineoa@medisin.uio.no (T.B. Opstad).

\section{Introduction}

With the global increase in life expectancy, age has become a major risk of most common diseases, such as cancer and cardiovascular disease (CVD). The impact of nutrition and caloric restriction (CR) on health span is evidenced, although results are mainly from animal models [1-3], and with differentiated impact depending on model organism, sex, diet and genetic factors [4,5]. In humans, studies on CR are mainly focused on weight 
reduction and different $\mathrm{CR}$ regimes [6,7]. The strive for healthier body-weight to reduce metabolic disturbances, such as type- 2 diabetes and CVD, is essential in preventive medicine. We have recently reported on beneficial effects of $\mathrm{CR}$ on weight loss and improvement in cardiometabolic risk factors after 1-year of intervention [8].

The favorable effects of nutrient restriction on cardiovascular ageing, seem partly mediated by the sirtuin system, shown from both animal and human studies [9]. With fasting, there is a molecular switch from liver-derived glucose to adipose tissue ketone bodies, which can regulate the activity of multiple proteins involved in health and longevity, including nicotinamide adenine dinucleotide $\left(\mathrm{NAD}^{+}\right)_{-}$molecules and sirtuins [10]. The NAD ${ }^{+}$- dependent deacetylase Sirtuin1 (SIRT1) is the most investigated among sirtuins and its function has been associated with longevity and protection from metabolic and chronic degenerative diseases [11,12]. SIRT1 is mainly located in cell nuclei of most tissues, including liver, pancreas, heart, skeletal muscle and adipose tissue, with the highest expression levels in leukocytes. Its expression has been shown downregulated in adipose tissue from obese women [13] and in peripheral blood mononuclear cells from subjects with insulin resistance and subclinical atherosclerosis [14]. SIRT1 deacetylates histones and nonhistone proteins and interacts with diverse transcription factors, thereby regulating diverse activities such as cell growth, cell survival, and NF- $\kappa$ B signaling with inhibited pro-inflammatory cytokine expression. SIRT1 expression seems further to be protective for vascular senescence through the activation of endothelial nitric oxide synthase and the inhibition of reactive oxygen species. SIRT1 is an important regulator of energy homeostasis in response to nutrient availability and can thus be activated by CR and further by certain polyphenols in fruits and vegetables, and by sirtuin-activating compounds (STACs) [15]. Clinical studies on CRs' effect on SIRT1 have hitherto involved small numbers and short-term interventions, reporting partly increased SIRT1 protein expression and elevated circulating levels $[16,17]$.

We therefore aimed to explore the effect of $C R$ on circulating SIRT1 levels in obese subjects enrolled to two different fasting regimes for 1 year. We hypothesized that CR will modify SIRT1 levels equally in both regimes and that the observed beneficial effects on cardiometabolic risk factors will be reflected in SIRT levels, enlightening more in depth the role of circulating SIRT1. As sex differences in SIRT1s' cardioprotective role have been suggested [18], results were stratified by gender.

\section{Methods}

\section{Study population}

The current study is a sub-study of a clinical trial that recruited 112 obese subjects with body mass index (BMI) $30-45 \mathrm{~kg} / \mathrm{m}^{2}$, aged $21-70$ years, into a randomized intervention trial on CR between 2015 and 2017 at Section of
Preventive Cardiology, Department of Endocrinology, Morbid Obesity and Preventive Medicine at Oslo University Hospital in Norway [8]. Block randomization was performed to secure equal distribution of sex and BMI in each intervention group. In addition to $\mathrm{BMI}>30 \mathrm{~kg} / \mathrm{m}^{2}$, inclusion criteria comprised waist circumference $\geq 94 / 80 \mathrm{~cm}$ (men/ women) and one additional metabolic syndrome component: triglycerides $\geq 1.7 \mathrm{mmoL} / \mathrm{l}$, HDL cholesterol $\leq 1.0 / 1.3$ (men/women), fasting glucose $\geq 5.6 \mathrm{mmol} / \mathrm{L}$, blood pressure $\geq 130 / 85 \mathrm{mmgHg}$ or use of antihypertensive medication. Weight stability $( \pm 3 \mathrm{~kg})$ was required during the last three months before inclusion. Exclusion criteria were treated diabetes, previous bariatric surgery, use of anti-obesity drugs or drugs affecting body-weight, eating disorders, alcohol or drug abuse, and psychiatric illness. Secondary obesity was an exclusion criterion, and optimal treatment of hypothyroidism was required. No participants had Cushing's disease (exclusion criteria if present) or polycystic ovarian syndrome (not an exclusion criterion) and genetic mutations causing obesity were not examined given their low prevalence in the population.

\section{Fasting regimes}

The subjects were randomized $1: 1$ to intermittent (ICR) or continuous energy restriction (CCR) groups for 1 year, as previously described [8]. In short, the ICR group consumed $400 / 600 \mathrm{kcal}$ (female/male) on two non-consecutive days with normal energy intake rest of the week, whereas the CCR group reduced their energy intake evenly for seven days. The weekly total energy intake was equal between intervention groups counted for individual total daily energy expenditure, and participants were advised to follow the general principles of a Mediterranean-type diet. All participants received individualized dietary plans, including educational material and diet counselling the first 6 months, and knowledge to cognitive behavioral methods to improve compliance. Adherence to diet regimens were encouraged by maintaining a consistent eating pattern and advising them about factors associated with weight loss maintenance. Self-reported physical activity was recorded, and all subjects were advised to not change their physical activity during the period, to avoid potential confounding. Dietary intake was recorded at baseline and 3 months using a diet tool according to the Norwegian Directorate of Health (http://www.kostholdsplanleggeren.no), and ten follow-up visits were conducted during 12 months of intervention. The study was approved by the local Regional Ethics Committee, and conducted according to the Declaration of Helsinki. Written informed consent was provided from all participants before enrolling in the study. The study is registered at www.clinicaltrials.gov NCT02480504.

\section{Clinical and laboratory measures}

Body weight and circumferences were measured after $10 \mathrm{~h}$ fast, blood pressure after 5 min of rest, and fasting blood samples were collected at inclusion and 12 months, as 
previously described [8]. Serum lipids, fasting glucose, HbA1c and C-reactive protein (CRP) were analyzed by conventional laboratory methods at Oslo University Hospital Clinical Chemistry Laboratory. Serum was prepared by centrifugation within $1 \mathrm{~h}$ at $2500 \mathrm{~g}$ for $10 \mathrm{~min}$ and kept frozen at $-80{ }^{\circ} \mathrm{C}$ until SIRT1 analysis, performed at baseline and after 1 year by the Human SIRT1 method from LSBio LifeSpan BioSciences, lnc (Seattle, USA). The interassay correlation of variation was $11.6 \%$, and SIRT1 was successfully analyzed in all available samples, with equal distribution of samples from each group before and after intervention on same ELISA plates to avoid differences due to assay variability.

\section{Statistical methods}

Due to skewed distribution of serum SIRT1 concentrations, mainly non-parametric statistics were used. Repeated measures and within-group differences were calculated by Wilcoxon Signed Rank test, R-related samples. As delta SIRT1 values (change from baseline levels to 12 months) were normally distributed, between-group differences were calculated by ANOVA repeated measures, mixed model. Spearman Rho was used for baseline correlations and correlation between delta values. Mann-Whitney $U$ test was used for differences in delta SIRT1 values in groups of delta BMI values dichotomized at median $\left(2.79 \mathrm{~kg} / \mathrm{m}^{2}\right)$. Sample size calculation was not performed, and numbers included in each intervention group are based on calculations in the initial study. Statistical calculations were performed using SPSS version 26 (SPSS Inc., Chicago, Illinois, USA). P-values $<0.05$ were defined as statistically significant.

\section{Results}

\section{Subject demographics}

During the study, three dropouts occurred in the CCR group versus four in the ICR group, resulting in 55 and 50 participants, respectively, completing the study. Baseline characteristics, including hormonal treatment in women, were equally distributed between the intervention regimes, except for higher triglycerides in the intermittent fasting group (Table 1). As previously reported, after 1 year, no statically significant difference in weight loss, BMI or waist and hip circumference reductions was observed between groups, with correspondingly 8.0 vs. $9.0 \mathrm{~kg}, 2.7$ vs. $3.2 \mathrm{~kg} / \mathrm{m}^{2}, 8.7$ vs. $9.6 \mathrm{~cm}$ and 6.8 vs. $7.5 \mathrm{~cm}$ in the ICR and CCR group, respectively [8]. These improvements were achieved mainly after 6 months of CR, and a slight but significant increase in weight and BMI was observed in the maintenance phase from 6 to 12 months in the ICR group. No between-group differences in changes in cardiometabolic risk factors were observed after 1 year. Diastolic blood pressure, HDL cholesterol, triglycerides and HbA1c (at 6 months) were improved in both groups, whereas systolic blood pressure was reduced in the continuous energy restriction group [8].

Serum samples fort SIRT1 analyses were available for 109 subjects at inclusion and 105 subjects at 12 months, 102 samples completing both visits (54 in the CCR group, 48 in the ICR group). The complete number $(n=102)$ was used in all statistical analyses, whereof 51 were women.

In women, significantly higher SIRT1 concentrations were observed in hormonal users $(\mathrm{n}=9)$ vs. non-users at baseline as follows: median (25, 75 percentiles) were 1229 $(790,1794)$ vs. $821(585,1102) \mathrm{pg} / \mathrm{mL}(\mathrm{p}=0.02)$.

\section{Baseline correlations between circulating SIRT1 and cardiometabolic risk factors}

In all, SIRT1 correlated significantly to BMI ( $p=0.019)$, heart rate $(\mathrm{p}=0.011)$ and CRP $(\mathrm{p}<0.001)$, with stronger correlations for all markers in women $(p<0.010$, all), in which SIRT1 also correlated to waist circumference $(p=0.033)$ (Table 3). When excluding results for the 9 women who were hormone users, the correlations remains statistically significant $(\mathrm{r}=.343-.526$, all $\mathrm{p}<0.05)$. In men, SIRT1 was significantly correlated to CRP.

\section{Change in circulating SIRT1}

SIRT1 concentrations at baseline and after 1 year are shown in Table 2, in total and according to CR regimens and sex. No significant differences in SIRT1 concentrations at baseline were observed between the randomized groups $(p=0.27)$, sex $(p=0.18)$, or in each randomized group stratified by gender ( $\mathrm{p}>0.1$ in both). After 1 year, no significant between-group differences were observed according to intervention regimen $(\mathrm{p}=0.98)$ and sex $(\mathrm{p}=0.41)$. In pairwise comparison, SIRT1 concentrations increased numerically in the total population $(\mathrm{p}=0.085)$, statistically significant in the ICR group $(\mathrm{p}=0.041)$ and in men following the ICR regimen $(p=0.016)$. When excluding results for the 9 women who were hormone users, similar non-statistically significant within group $\mathrm{p}$ values were observed in women ( $\mathrm{p}=0.57)$, in women on CCR $(\mathrm{p}=0.82)$ and in women on ICR $(\mathrm{p}=0.33)$.

\section{Correlations between change in SIRT1 concentrations (delta values) and changes in anthropometric levels}

In total, and in the different CR regimens, delta SIRT1 values after 1 year were not correlated to changes in anthropometric levels. Stratified by gender, delta SIRT1 values in women correlated to changes in body weight $(\mathrm{r}=.295)$, BMI $(\mathrm{r}=.298)$ and waist circumference $(\mathrm{r}=.321), \mathrm{p}<0.05$ for all (Table 4$)$, with stronger correlation to BMI changes in women in the ICR group $(\mathrm{r}=.424, \mathrm{p}=0.049)$. In men, improvements in BMI were inversely correlated to delta SIRT1 values, only in the ICR group $(r=-.396, p=0.045)$. No significant correlations were observed in the CCR group, stratified by gender. 
Table 1 Baseline cardiometabolic risk factors according to fasting regimens.

\begin{tabular}{|c|c|c|c|c|}
\hline & All & $\begin{array}{l}\text { Continuous CR } \\
\mathrm{n}=54\end{array}$ & $\begin{array}{l}\text { Intermittent CR } \\
\mathrm{n}=48\end{array}$ & p-value \\
\hline Age & $48.8(10.5)$ & $48.0(11.1)$ & $50(10)$ & 0.40 \\
\hline Men/Women & $51 / 51$ & $25 / 29$ & $26 / 22$ & 0.43 \\
\hline BMI, $\mathrm{kg} / \mathrm{m}^{2}$ & $35(32,38)$ & $35(32,38)$ & $35(32,38)$ & 0.84 \\
\hline Body weight, kg & $108(16)$ & $107(16)$ & $109(17)$ & 0.36 \\
\hline Systolic blood pressure $\mathrm{mmHg}$ & $129(14)$ & $128(14)$ & $129(14)$ & 0.82 \\
\hline Diastolic blood pressure $\mathrm{mmHg}$ & $87(9)$ & $86(9)$ & $88(8)$ & 0.25 \\
\hline Heart rate/minute & $68(9)$ & $68(8)$ & $68(11)$ & 0.92 \\
\hline Glucose, $\mathrm{mmol} / \mathrm{L}$ & $5.79(1.00)$ & $5.73(0.67)$ & $5.86(1.29)$ & 0.52 \\
\hline $\mathrm{HbA} 1 \mathrm{c}, \%$ & $5.55(0.65)$ & $5.48(0.55)$ & $5.63(0.75)$ & 0.27 \\
\hline Total cholesterol, mmol/L & $5.06(0.89)$ & $5.14(0.87)$ & $4.98(0.92)$ & 0.36 \\
\hline HDL-cholesterol, mmol/L & $1.18(0.30)$ & $1.17(0.26)$ & $1.20(0.34)$ & 0.72 \\
\hline LDL-cholesterol, mmol/L & $3.39(0.85)$ & $3.49(0.84)$ & $3.26(0.86)$ & 0.19 \\
\hline $\begin{array}{l}\text { Triglycerides, mmol/L, median levels } \\
\text { (25, 75percentiles) }\end{array}$ & $1.62(1.20,2.04)$ & $1.42(1.15,1.89)$ & $1.71(1.29,2.52)$ & 0.040 \\
\hline $\mathrm{CRP}, \mathrm{mg} / \mathrm{L}$ & $3.83(3.57)$ & $4.45(3.86)$ & $3.15(3.12)$ & 0.068 \\
\hline Antidiabetic drugs, n (\%) & $6(6)$ & $5(5)$ & $1(1)$ & 0.28 \\
\hline Statins, n (\%) & $17(17)$ & $7(7)$ & $10(10)$ & 0.59 \\
\hline Antihypertension drugs, n (\%) & $39(38)$ & $22(22)$ & $17(17)$ & 0.74 \\
\hline Female hormonal treatment ${ }^{a}$ & $9(18)$ & $5(10)$ & $4(8)$ & 0.94 \\
\hline Use of alcohol, n (\%) & $80(78)$ & $42(41)$ & $38(37)$ & 0.64 \\
\hline Smokers, n (\%) & $7(9)$ & $3(3)$ & $4(4)$ & 0.58 \\
\hline
\end{tabular}

Mean (SD) values are shown, if not otherwise stated. CR; caloric restriction, BMI; body mass index, HbA1c; glycated hemoglobin, HDL; high density lipoprotein, LDL; low density lipoprotein, CRP; C-reactive protein, p-values indicate differences between intervention groups (Mann-Whitney U test). Bold text represent p-values $<0.05$.

${ }^{a}$ Out of 9, 6 used oral contraceptives, 2 women used hormone replacement therapy and 1 used hormones to treat acne.

Table 2 Change in circulating SIRT1 from baseline to 1 year in total and according to assignment to continuous or intermittent CR and sex.

\begin{tabular}{|c|c|c|c|c|}
\hline SIRT1 $\mathrm{pg} / \mathrm{mL}$ & Baseline & 12 months & $\begin{array}{l}\mathrm{p} \text {-value } \\
\text { within-groups }^{\mathrm{a}}\end{array}$ & $\begin{array}{l}\text { p-value } \\
\text { between-groups }\end{array}$ \\
\hline Total & $832(574,1120)$ & $898(623,1238)$ & 0.085 & \\
\hline Continuous CR & $850(597,1121)$ & $905(620,1096)$ & 0.61 & 0.98 \\
\hline Intermittent CR & $762(530,1135)$ & $884(624,1285)$ & 0.041 & \\
\hline Men & $758(554,992)$ & $870(621,1245)$ & 0.062 & 0.41 \\
\hline Women & $873(597,1221)$ & $925(651,1236)$ & 0.56 & \\
\hline \multicolumn{5}{|l|}{ Continuous CR } \\
\hline Men & $841(592,1150)$ & $924(613,1300)$ & 0.72 & 0.72 \\
\hline Women & $854(601,1133)$ & $903(634,1038)$ & 0.64 & \\
\hline \multicolumn{5}{|l|}{ Intermittent CR } \\
\hline Men & $633(524,926)$ & $820(623,1250)^{*}$ & 0.016 & 0.16 \\
\hline Women & $908(559,1334)$ & $1085(644,1347)$ & 0.45 & \\
\hline
\end{tabular}

CR; caloric restriction.

Bold text represent p-values $<0.05$.

${ }^{a}$ Wilcoxon Signed Rank test, R-related samples.

b Repeated measures ANOVA, mixed model.

Notably, improvements in BMI after 1 year was nonstatistically higher in women vs. in men $\left(3.0 \mathrm{~kg} / \mathrm{m}^{2}\right.$ vs. $2.54 \mathrm{~kg} / \mathrm{m}^{2}, \mathrm{p}=0.12$ ). To further explore the observed associations between BMI and SIRT1, improvements in BMI after 1year were dichotomized at median $\left(2.8 \mathrm{~kg} / \mathrm{m}^{2}\right)$, to assess the distribution of change in SIRT1 values accordingly (Fig. 1). In all, and in the different intervention regimens, no significant difference in SIRT1 changes was observed according to BMI groups. Stratified by gender, delta SIRT1 values increased significantly in women when change in BMI was < median and decreased with change in BMI above median ( $p=0.017$, for difference betweengroups). Stratified by sex in each CR regimen, the difference in delta SIRT1 values between BMI groups was borderline significant in women in the ICR group $(\mathrm{p}=0.08)$. Overall, limited BMI changes $\left(<2.8 \mathrm{~kg} / \mathrm{m}^{2}\right)$ induced an increase in SIRT1 concentrations, significant in the total population $(\mathrm{p}=0.030)$ and in women $(\mathrm{p}=0.021)$, whereas BMI changes $>$ median increased SIRT1 concentrations significantly in men following the ICR regime $(\mathrm{p}=0.013)$.

\section{Correlations between change (delta) in SIRT1 values and observed changes in cardiometabolic risk factors}

In the main study, within-group improvements were observed in blood pressure, HDL cholesterol, triglycerides and HbA1c (at 6 months) after 1 year of CR, but with no between-group differences [8]. SIRT1 concentrations did not correlate to these changes after 1 year, except for a 
Table 3 Baseline correlations between SIRT1 and cardiometabolic risk factors in the total population and according to sex.

\begin{tabular}{|c|c|c|c|c|c|c|c|c|c|c|c|c|c|c|}
\hline & BMI & Weight & Waist circ. & Hip circ. & SBP & DBP & Heart rate & f. Glucose & HbA1c & Chol. & HDL c. & LDL c. & TG & CRP \\
\hline \multirow[t]{2}{*}{ All } & .232 & .086 & .192 & .166 & -.015 & -.046 & .251 & -.039 & .140 & -.089 & -.028 & -.079 & .058 & .450 \\
\hline & .019 & .39 & .053 & .096 & .29 & .65 & .011 & .70 & .16 & .37 & .78 & .43 & .57 & $<.001$ \\
\hline \multirow[t]{2}{*}{ Men } & .112 & .153 & .192 & .09 & -.076 & -.055 & .111 & -.083 & .098 & -.105 & -.022 & -149 & .111 & .419 \\
\hline & .43 & .29 & .18 & .53 & .60 & .70 & .44 & .56 & .50 & .46 & .88 & 30 & .44 & .002 \\
\hline \multirow[t]{2}{*}{ Female } & .368 & .205 & .300 & .191 & -.06 & ,014 & .400 & .029 & .159 & -.104 & -.089 & -.058 & .019 & .482 \\
\hline & .008 & .15 & .033 & .18 & .68 & .93 & .004 & .84 & .27 & .47 & .54 & .69 & .90 & $<.001$ \\
\hline
\end{tabular}

Spearman Rho correlations are shown with italicized p-values underneath.

Bold text indicate statistically significant correlations with p-values $<0.05$.

BMI; body mass index, Waist/Hip circ; circumference, SBP, systolic blood pressure, DBP; diastolic blood pressure, f. Glucose; fasting glucose levels, Chol; cholesterol, HDL c.; high density lipoprotein cholesterol, LDL c.; low density lipoprotein cholesterol, TG; triglycerides, CRP; C-reactive protein.

moderate correlation between delta SIRT1 values and change in diastolic blood pressure after 1 year in women following the ICR $(\mathrm{r}=.496, \mathrm{p}=0.02, \mathrm{n}=22)$.

\section{Discussion}

The main findings in the present study were that 1) After 1 year, no significant between-group differences in SIRT1 levels were observed according to regimen and sex 2) In intermittent CR group, Sirtuin1 concentrations increased in the total population and in men 3) In women, improvement in BMI after 1 year correlated to Sirtuin1 changes, with stronger correlation in the intermittent CR group and 4) In men, improvements in BMI correlated inversely to Sirtuin 1 changes in the intermittent CR group.

Table 4 Correlations between changes (delta, $\Delta$ ) in SIRT1 concentration and anthropometric levels after 1 year of intervention in all and according to intervention regimen and sex.

\begin{tabular}{|c|c|c|c|c|}
\hline & $\Delta \mathrm{BMI}$ & $\Delta$ Weight & $\Delta$ Waist circ. & $\Delta$ Hip circ \\
\hline \multirow[t]{2}{*}{ All } & .086 & .069 & .140 & .057 \\
\hline & .39 & .49 & .16 & .57 \\
\hline \multirow[t]{2}{*}{ CCR } & .129 & .112 & .200 & -.014 \\
\hline & .35 & .42 & .15 & .92 \\
\hline \multirow[t]{2}{*}{ ICR } & -.010 & -.030 & .058 & .109 \\
\hline & .95 & .84 & .70 & .46 \\
\hline \multirow[t]{2}{*}{ Men } & -.193 & -.159 & -.068 & -.178 \\
\hline & .18 & .26 & .63 & .21 \\
\hline \multirow[t]{2}{*}{ Women } & .298 & .295 & .321 & .260 \\
\hline & .034 & .035 & .022 & .065 \\
\hline \multicolumn{5}{|l|}{ CCR } \\
\hline \multirow[t]{2}{*}{ Men } & .033 & .050 & .242 & -.190 \\
\hline & .88 & .81 & .25 & .36 \\
\hline \multirow[t]{2}{*}{ Women } & .176 & .164 & .142 & .110 \\
\hline & .36 & .40 & .46 & .57 \\
\hline \multicolumn{5}{|l|}{ ICR } \\
\hline \multirow[t]{2}{*}{ Men } & -.396 & -.334 & -.371 & -.197 \\
\hline & .045 & .095 & .062 & .33 \\
\hline \multirow[t]{2}{*}{ Women } & .424 & .414 & .417 & .423 \\
\hline & .049 & .056 & .054 & .050 \\
\hline
\end{tabular}

Spearman Rho correlations are shown with italicized p-values underneath.

Bold text indicate statistically significant correlations with p-values $<0.05$.

BMI; body mass index, Waist/Hip circ.; Waist/Hip circumference, CCR and ICR; continuous and intermittent caloric restriction, respectively.
So far, the implication of CR type in humans is still uncertain [19]. The present study investigating CR in obese subjects indicates that the explicit effects on SIRT1 are related to type of CR regimen, sex and BMI, to our knowledge not previously reported. ICR induced higher circulating SIRT1 concentration, especially in men, in which increased SIRT1values were inversely related to improvements in BMI. In women, SIRT1 changes was BMIdependent, with significantly increased and numerically decreased SIRT1 concentrations according to below or above median BMI loss, with significant correlation between SIRT1-and BMI changes especially after 1 year with ICR.

Both intervention groups reduced weight, BMI, and hip circumferences similarly after 1 year, whereas sexstratified, the reduction in BMI was more advanced in women. In a study by Harvie et al. obese young women lost more body fat with ICR than CCR after 4 months of intervention [20], partly supporting our findings. Experimental and animal studies have also indicated that fasting with ICR reduces body weight and improves metabolic risk factors to a greater extent than CCR [21], although contradictory reported $[7,22]$. In the present study, the fasting component being more prominent in ICR seems to have affected SIRT1 concentrations more than CCR, independent of initial BMI, but influenced by sex and BMI improvements. Subjects in the ICR group did also report stronger feelings of hunger throughout the study [8]. As many of the intervened subjects in our study were severely obese, stronger weight and BMI reduction might be required to achieve more clear effects on SIRT1. We have previously reported a positive correlation between circulating SIRT1and BMI in healthy subjects [23], contradictory reported by others [24]. In the same population, we observed SIRT1 leukocyte expression to be inversely associated with BMI, as also found by others [25]. Circulating SIRT1 has been reported to be elevated in frailty older adults, including those with weight loss [26], further complicating the picture.

The observed sex-related SIRT1 changes cannot ignore the potential influence of estrogen and the use of oral contraceptives and hormone replacement therapy, although a limited number were treated with hormones. Underlying mechanisms of suggested sex differences in 

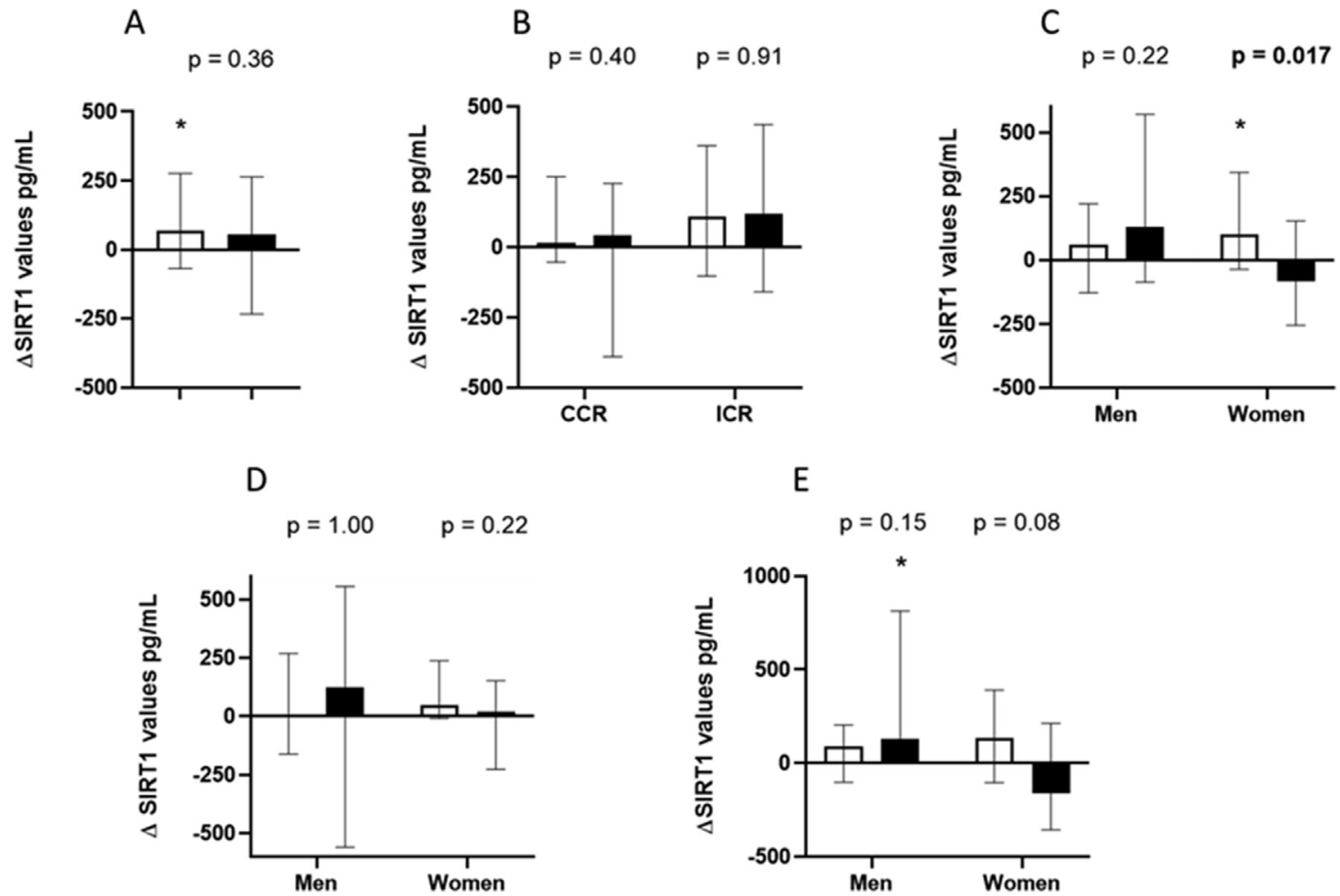

Figure 1 Change in SIRT1 values according to BMI improvements after 1 year, categorized at median $\left(2.8 \mathbf{~ k g} / \mathbf{m}^{2}\right)$. White and black columns indicate delta SIRT1 values with BMI improvements below and above median, respectively. A) in the total population, B) according to intervention regime, C) according to sex, D) CCR stratified by sex, E) ICR stratified by sex. p-values indicate differences between-groups categorized at median BMI changes (Mann-Whitney U test), " represents $\mathrm{p}<0.05$ for within-group differences (Wilcoxon Signed Rank test, R-related samples). CCR; continuous caloric restriction, ICR; intermittent caloric restriction.

atherosclerotic cardiovascular disease have recently been revisited, including SIRT1 and estrogen [18]. Genetic SIRT1 polymorphisms, previously linked to BMI and sever obesity [27], may also have influenced circulating SIRT1 levels, although sex-dimorphism in their frequency is not known. Women may also be more receptive to healthpromoting life-style adjustments, and as women may pose greater control over mitochondrial function and turnover, a better response to diet and environment in females has been suggested [28,29]. CR-induced SIRT1 changes may also be time-dependent, suggesting women to respond faster with metabolic alterations than men.

Baseline analyses showed that circulating SIRT1 was significantly correlated to CRP, indicating circulating SIRT1 to be related to inflammation, as we previously have reported $[23,28]$, and the present obese population most likely suffers from pro-inflammatory metabolic disturbances. We have previously reported on circulating SIRT1s positive association with known cardiovascular risk factors like cigarette smoking, in addition to BMI, in healthy subjects [23]. In that particular study we also demonstrated that SIRT1 gene-expression was inversely correlated to circulating SIRT1 [23]. In patients with stable coronary artery disease, we further observed that genetically expressed SIRT1 was inversely associated with inflammatory markers, such as interleukin (IL) -6, IL-18 and INF-y, especially in females [28], as also observed by others in patients with concomitant type 2 diabetes and CAD [30]. Recently, a sex-specific downregulation of SIRT1in aging human female hearts was associated with a significant increase in pro-inflammatory cytokines, as well as NF-kB upregulation, indicating a closer relationship of SIRT1 and inflammation in women compared to men [31], again underpinning potential sex-differences in SIRT1.

All together, the present study indicates that SIRT1 changes after CR are limited associated with improvement in cardiometabolic risk factors. A decrease in diastolic blood pressure after 1 year was associated with reduction in SIRT1 concentration in women, supporting the suggestion that low rather than high circulating SIRT1 levels are beneficial. SIRT1 activity and diastolic blood pressure were also reported to be correlated exclusively in women in healthy adults [32], substantiating our results. One may therefore question the impact of elevated circulating SIRT1 levels, being potentially upregulated when its leukocyte gene-expression is reduced, as previously observed, and vice versa. SIRT1s' action is mainly located in the nucleus and the amount of SIRT1 released into the circulation may eventually reflect compensatory up- or down-regulated mechanisms from other cell-types than leukocytes under certain clinical conditions. From the present study it might be speculated that BMI is a driver of circulating SIRT1 in women, although other have suggested waist circumference to be determinant, independent of sex [24]. 


\section{Limitations}

The main limitation in our study was lack of SIRT1 geneexpression and eventually SIRT1 activity data, which could have enlightened more in depth the impact of different CR regime and sex on SIRT1. Additionally also investigating other sirtuins could have given a broader picture on CRs' effects on the sirtuin system. Although being a CR intervention study, with relatively high number, subgroups are small, requiring caution in interpreting results. It should also be emphasized that the study population is rather special, being obese, thus the results may be influenced by dysregulated hormones, other than estrogens, insulin sensitivity and adipose tissue inflammation. The strength in our study is the 1-year duration of intervention.

In conclusion, the change in circulating SIRT1 concentration after CR was related to type of regime, sex and BMI, supporting individualized dietary intervention, provided clinical relevance of SIRT1 on health benefits. The ICR regimen seems to have affected SIRT1 to a stronger extent than CCR. The impact of measuring circulating SIRT1 needs further investigation.

\section{Funding}

This work was founded by Stein Erik Hagen Foundation for Clinical Heart Research, Oslo Norway and the Norwegian Health Association. The funding organizations were not represented in the writing group and had no role in study design, data collection, analysis, Decision to publish or preparation of the manuscript.

\section{Declaration of competing interest}

None.

\section{Acknowledgements}

We especially want to thank Eli Heggen for her support and assistance in making blood samples available. In the main study, Merethe Helgeland is acknowledged for dietary counseling, Sasa Dusanov for medical examination and RMR measurements, Eli Heggen and Tor Ole Klemsdal for medical examination and Ragnhild Kleve and Lise Bergengen for assistance with study procedure.

\section{References}

[1] Mathers JC. Impact of nutrition on the ageing process. Br J Nutr 2015;113(Suppl):S18-22. https://doi.org/10.1017/s0007114514003 237.

[2] Simons MJ, Koch W, Verhulst S. Dietary restriction of rodents decreases aging rate without affecting initial mortality rate - a meta-analysis. Aging Cell 2013;12(3):410-4. https://doi.org/10. 1111/acel.12061.

[3] Colman RJ, Beasley TM, Kemnitz JW, Johnson SC, Weindruch R, Anderson RM. Caloric restriction reduces age-related and all-cause mortality in rhesus monkeys. Nat Commun 2014;5:3557. https: //doi.org/10.1038/ncomms4557.
[4] Nakagawa S, Lagisz M, Hector KL, Spencer HG. Comparative and meta-analytic insights into life extension via dietary restriction. Aging Cell 2012;11(3):401-9. https://doi.org/10.1111/j.1474-9726. 2012.00798.x.

[5] Mattison JA, Roth GS, Beasley TM, Tilmont EM, Handy AM, Herbert RL, et al. Impact of caloric restriction on health and survival in rhesus monkeys from the NIA study. Nature 2012;489(7415):318-21. https://doi.org/10.1038/nature11432.

[6] Parvaresh A, Razavi R, Abbasi B, Yaghoobloo K, Hassanzadeh A, Mohammadifard N, et al. Modified alternate-day fasting vs. calorie restriction in the treatment of patients with metabolic syndrome: a randomized clinical trial. Compl Ther Med 2019;47:102187. https: //doi.org/10.1016/j.ctim.2019.08.021.

[7] Headland M, Clifton PM, Carter S, Keogh JB. Weight-loss outcomes: a systematic review and meta-analysis of intermittent energy restriction trials lasting a minimum of 6 months. Nutrients $2016 ; 8(6)$. https://doi.org/10.3390/nu8060354.

[8] Sundfor TM, Svendsen M, Tonstad S. Effect of intermittent versus continuous energy restriction on weight loss, maintenance and cardiometabolic risk: a randomized 1-year trial. Nutr Metabol Cardiovasc Dis : Nutr Metabol Cardiovasc Dis 2018;28(7):698-706. https://doi.org/10.1016/j.numecd.2018.03.009.

[9] Guarente L. Sirtuins, aging, and metabolism. Cold Spring Harbor Symp Quant Biol 2011;76:81-90. https://doi.org/10.1101/sqb.2011. 76.010629.

[10] de Cabo R, Mattson MP. Effects of intermittent fasting on health, aging, and disease. N Engl J Med 2019;381(26):2541-51. https: //doi.org/10.1056/NEJMra1905136.

[11] Saunders LR, Verdin E. Sirtuins: critical regulators at the crossroads between cancer and aging. Oncogene 2007;26(37): 5489-504. https://doi.org/10.1038/sj.onc.1210616.

[12] Haigis MC, Sinclair DA. Mammalian sirtuins: biological insights and disease relevance. Annu Rev Pathol 2010;5:253-95. https: //doi.org/10.1146/annurev.pathol.4.110807.092250.

[13] Pedersen SB, Ølholm J, Paulsen SK, Bennetzen MF, Richelsen B. Low Sirt1 expression, which is upregulated by fasting, in human adipose tissue from obese women. Int J Obes 2008;32(8):1250-5. https://doi.org/10.1038/ijo.2008.78.

[14] de Kreutzenberg SV, Ceolotto G, Papparella I, Bortoluzzi A, Semplicini A, Dalla Man C, et al. Downregulation of the longevity-associated protein sirtuin 1 in insulin resistance and metabolic syndrome: potential biochemical mechanisms. Diabetes 2010;59(4):1006-15. https://doi.org/10.2337/db09-1187.

[15] Heiss C, Spyridopoulos I, Haendeler J. Interventions to slow cardiovascular aging: dietary restriction, drugs and novel molecules. Exp Gerontol 2017. https://doi.org/10.1016/j.exger.2017.06.015.

[16] Lopez-Domenech S, Abad-Jimenez $Z$, Iannantuoni $F$, de Marañón AM, Rovira-Llopis S, Morillas C, et al. Moderate weight loss attenuates chronic endoplasmic reticulum stress and mitochondrial dysfunction in human obesity. Mol Metab 2019;19: 24-33. https://doi.org/10.1016/j.molmet.2018.10.005.

[17] Mansur AP, Roggerio A, Goes MFS, Avakian SD, Leal DP, Maranhão RC, et al. Serum concentrations and gene expression of sirtuin 1 in healthy and slightly overweight subjects after caloric restriction or resveratrol supplementation: a randomized trial. Int J Cardiol 2017;227:788-94. https://doi.org/10.1016/j.ijcard.2016. 10.058.

[18] Shimabukuro M. SIRT1 and gender differences in atherosclerotic cardiovascular disease. J Atherosclerosis Thromb 2020;27(1): 8-10. https://doi.org/10.5551/jat.ED119.

[19] Sundfør TM, Svendsen M, Tonstad S. Intermittent calorie restriction-a more effective approach to weight loss? Am J Clin Nutr 2018;108(5):909-10. https://doi.org/10.1093/ajcn/nqy288.

[20] Harvie M, Wright C, Pegington M, McMullan D, Mitchell E, Martin B, et al. The effect of intermittent energy and carbohydrate restriction $\mathrm{v}$. daily energy restriction on weight loss and metabolic disease risk markers in overweight women. $\mathrm{Br} \mathrm{J}$ Nutr 2013;110(8):1534-47. 1017/s0007114513000792.

[21] Varady KA, Hellerstein MK. Alternate-day fasting and chronic disease prevention: a review of human and animal trials. Am J Clin Nutr 2007;86(1):7-13. https://doi.org/10.1093/ajcn/86.1.7.

[22] Harris L, McGarty A, Hutchison L, Ells L, Hankey C. Short-term intermittent energy restriction interventions for weight 
management: a systematic review and meta-analysis. Obes Rev 2018;19(1):1-13. https://doi.org/10.1111/obr.12593.

[23] Opstad TB, Kalstad AA, Holte KB, Berg TJ, Solheim S, Arnesen H, et al. Shorter leukocyte telomere lengths in healthy relatives of patients with coronary heart disease. Rejuvenation Res 2020:2258. https://doi.org/10.1089/rej.2019.

[24] Mariani S, di Giorgio MR, Martini P, Persichetti A, Barbaro G, Basciani S, et al. Inverse association of circulating SIRT1 and adiposity: a study on underweight, normal weight, and obese patients. Front Endocrinol 2018;9:449. https: //doi.org/10.3389/fendo.2018.00449.

[25] Stefanowicz M, Nikołajuk A, Matulewicz N Karczewska-Kupczewska M. Adipose tissue, but not skeletal muscle, sirtuin 1 expression is decreased in obesity and related to insulin sensitivity. Endocrine 2018;60(2):263-71. https://doi. org/10.1007/s12020-018-1544-1.

[26] Ma L, Niu H, Sha G, Zhang Y, Liu P, Li Y. Serum SIRT1 is associated with frailty and adipokines in older adults. J Nutr Health Aging 2019;23(3):246-50. https://doi.org/10.1007/s12603-018-1149-7.

[27] Clark SJ, Falchi M, Olsson B, Jacobson P, Cauchi S, Balkau B, et al. Association of sirtuin 1 (SIRT1) gene SNPs and transcript expression levels with severe obesity. Obesity 2012;20(1):178-85. https://doi.org/10.1038/oby.2011.200.
[28] Opstad TB, Kalstad AA, Pettersen AA, Arnesen H, Seljeflot I. Novel biomolecules of ageing, sex differences and potential underlying mechanisms of telomere shortening in coronary artery disease. Exp Gerontol 2019;119:53-60. https://doi.org/10.1016/j.exger. 2019.01.020.

[29] Tower J. Sex-specific gene expression and life span regulation. Trends Endocrinol Metabol: TEM (Trends Endocrinol Metab) 2017; 28(10):735-47. https://doi.org/10.1016/j.tem.2017.07.002.

[30] Li Y, Ni J, Guo R, Li W. In patients with coronary artery disease and type 2 diabetes, SIRT1 expression in circulating mononuclear cells is associated with levels of inflammatory cytokines but not with coronary lesions. BioMed Res Int 2016:8734827. https://doi.org/10. $1155 / 2016 / 8734827$.

[31] Barcena de Arellano ML, Pozdniakova S, Kühl AA, Baczko I, Ladilov Y, Regitz-Zagrosek V. Sex differences in the aging human heart: decreased sirtuins, pro-inflammatory shift and reduced anti-oxidative defense. Aging 2019;11(7):1918-33. https://doi. org/10.18632/aging.101881.

[32] Lee HJ, Yang SJ. Aging-related correlation between serum sirtuin 1 activities and basal metabolic rate in women, but not in men. Clin Nutr Res 2017;6(1):18-26. https://doi.org/10.7762/cnr.2017.6.1.18. 